02

\title{
Оценка соотношения энергий излучения Вавилова-Черенкова и катодолюминесценции, возбуждаемых электронным пучком в алмазе
}

\author{
(C) Е.Х. Бакшт, К.П. Артёмов, А.Г. Бураченко, В.Ф .Тарасенко \\ Институт сильноточной электроники Сибирского отделения РАН, \\ 634055 Томск, Россия \\ e-mail: beh@loi.hcei.tsc.ru
}

Поступила в редакцию 20.03.2019 г.

В окончательной редакции 16.04.2019 г.

Принята к публикации 23.05.2019 г.

\begin{abstract}
Проведены оценки соотношения энергий излучения Вавилова-Черенкова и катодолюминесценции, возбуждаемых электронным пучком в алмазных образцах, с учетом рассеяния электронов в этих образцах, распределения электронов пучка по энергиям, ионизационных потерь энергии электронов и дисперсии показателя преломления алмаза. Приведены экспериментальные результаты по измерению спектральных характеристик свечения образцов природного и синтетического алмаза под действием электронного пучка субнаносекундной длительности с энергией электронов до $200 \mathrm{keV}$. Показано, что основная доля излучения алмазных образцов в области 240-750 nm под действием электронного пучка с энергией электронов до $200 \mathrm{keV}$ принадлежит катодолюминесценции.
\end{abstract}

Ключевые слова: алмазы, излучение Вавилова-Черенкова, катодолюминесценция.

DOI: $10.21883 /$ OS.2019.10.48370.120-19

\section{Введение}

Взаимодействие электронных пучков с прозрачными твердыми телами связано с возбуждением свечения в этих телах. Анализ свечения позволяет получать информацию как о характеристиках облучаемых образцов, так и о параметрах самих электронных пучков. Поэтому методы, основанные на анализе свечения твердого тела, находят широкое практическое применение.

При движении электронов в прозрачной среде со скоростью, большей скорости света в этой среде, электронный пучок возбуждает в этой среде излучение Вавилова-Черенкова (ИВЧ), физическая природа которого отличается от физической природы люминесценции. На этом явлении основан целый класс детекторов заряженных частиц - черенковские детекторы. Эти детекторы в последние годы используют и для определения параметров пучков убегающих электронов в установках токамак [1-4]. Черенковские детекторы такого типа, как правило, состоят из радиатора (среды, в которой возникает ИВЧ), ФЭУ и оптической системы, передающей излучение от радиатора к ФЭУ. При этом одним из перспективных материалов для радиаторов в подобных детекторах считается алмаз.

Убегающие электроны возникают при работе токамаков в различных режимах, а также при срыве плазмы [2]. Они могут иметь энергию как в единицы-десятки МэВ, так и сотни-десятки кэВ [1-4] и оказывают влияние на поведение плазмы, поскольку могут переносить значительную часть плазменного тока, а также могут вызывать эрозию стенок вакуумной камеры, что приводит к появлению вредных примесей, затрудняющих получение управляемой термоядерной реакции, и к повреждению стенок камеры. Поэтому очень важно контролировать появление потоков убегающих электронов в токамаках и определять их параметры с помощью различных датчиков, в том числе и черенковских детекторов.

При облучении электронным пучком прозрачных образцов электронный пучок также возбуждает люминесценцию этих образцов, которую принято называть катодолюминесценцией. Если пучок импульсный, то наблюдается импульсная катодолюминесценция (ИКЛ) [5]. В процессе определения параметров электронных потоков с энергией электронов в диапазоне десяткисотни кэВ с помощью черенковских детекторов в радиаторе, кроме ИВЧ, может также возникать сильная катодолюминесценция [6]. Поэтому необходимо знать количественное соотношение люминесценции и ИВЧ в радиаторе детектора.

В настоящей работе с применением математического моделирования проведены оценки соотношения ИКЛ и ИВЧ в алмазных образцах при облучении их электронным пучком с максимальной энергией электронов в спектре $\sim 200 \mathrm{keV}$.

\section{Экспериментальная установка и методики измерений}

Для возбуждения ИВЧ и ИКЛ в алмазных образцах была создана экспериментальная установка на основе высоковольтного генератора РАДАН-220 (напряжение холостого хода $\sim 250 \mathrm{kV}$, длительность импульса напряжения в случае согласованной нагрузки $\sim 2 \mathrm{~ns}$ на 
полувысоте, фронт импульса напряжения $\sim 0.5 \mathrm{~ns}$ по уровню 0.1-0.9) [7]. Использовался газовый диод, в котором генерировался пучок убегающих электронов длительностью $\sim 0.1 \mathrm{~ns}$ на полувысоте с плотностью тока $\sim 1 \mathrm{~A} / \mathrm{cm}^{2}$. Электронный пучок выводился через анодную Ті-фольгу толщиной $13 \mu \mathrm{m}$. Распределение электронов пучка по энергиям приведено на рис. 1. Видно, что более $95 \%$ электронов пучка имеют энергию, достаточную для возбуждения ИВЧ в алмазе (> $50 \mathrm{keV})$. Использовались плоскопараллельные пластинки природного и синтетического (CVD) алмаза IІа-типа толщиной $0.25 \mathrm{~mm}$ и $0.5 \mathrm{~mm}$ и с характерными поперечными размерами 5 и $10 \mathrm{~mm}$ соответственно. В экспериментах образцы располагались так, что их плоскопараллельные грани были перпендикулярны направлению распространения электронного пучка. Экспериментальная установка и сами эксперименты подробно описаны в [6].

Спектры излучения алмазных образцов под действием электронного пучка, а также спектры их пропускания регистрировались с помощью спектрометра OceanOptics $\mathrm{HR} 2000$ + ES (диапазон длин волн - 190-1100 nm, разрешение $\sim 0.9 \mathrm{~nm})$ с известной спектральной чувствительностью.

Схема регистрации ИВЧ представлена на рис. 2. Для более интенсивного воздействия пучка убегающих электронов на исследуемый образец использовалась диэлектрическая трубка, которая одевалась на трубчатый катод. Благодаря этой трубке удалось улучшить равномерность плотности электронов на образце. С помощью линзы (фокусное расстояние $F=75 \mathrm{~mm}$, диаметр $D=51.5 \mathrm{~mm}$ ) строилось изображение на щели монохроматора МДР-23 (решетка - $1200 \mathrm{~mm}^{-1}$, величина обратной линейной дисперсии - $13 \AA / \mathrm{mm}$, ширина входной и выходной щелей монохроматора $400 \mu \mathrm{m})$. За монохроматором располагался ФЭУ Н773210 Hamamatsu (диапазон длин волн - 185-900 nm, диапазон изменения чувствительности ФЭУ $-10^{3}-10^{7}$, время нарастания переходной характеристики - $2.2 \mathrm{~ns}$ ).

Линза располагалась на одинаковом расстоянии от образца и щели монохроматора $(\sim 150 \mathrm{~mm})$. Сигналы

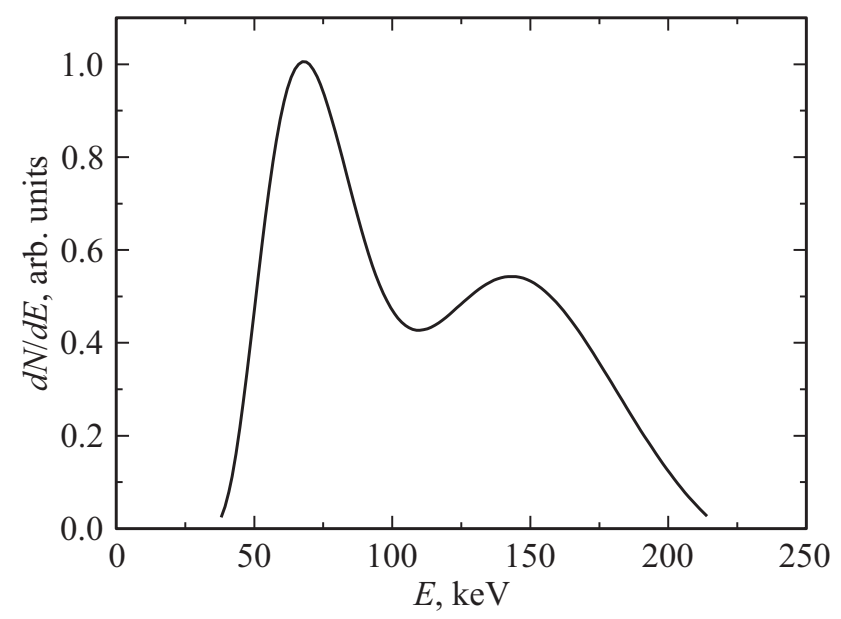

Рис. 1. Спектр электронного пучка.

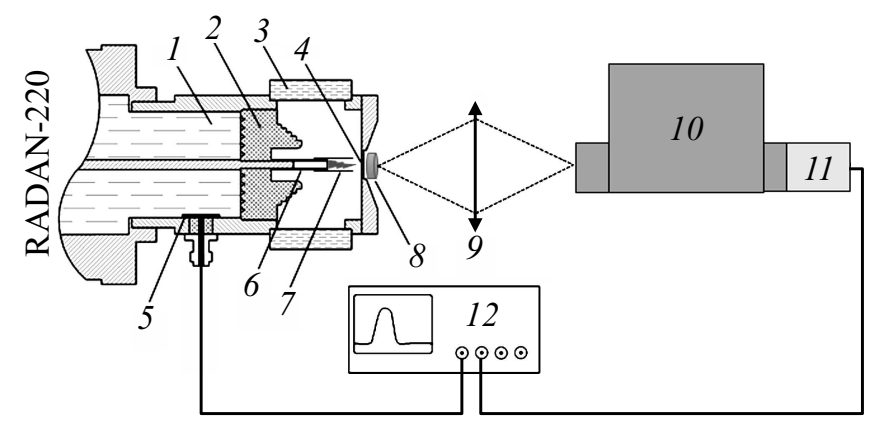

Рис. 2. Схема регистрации ИВЧ. 1 - трансформаторное масло, 2 - изолятор, 3 - выходные окна, 4 - анод, 5 делитель напряжения, 6 - катод, 7 - диэлектрическая трубка, 8 - исследуемый образец, $9-$ линза, $10-$ монохроматор, 11 - ФЭУ, 12 - осциллограф.

с ФЭУ регистрировались с помощью цифрового осциллографа Tektronix TDS3054B (полоса пропускания $0.5 \mathrm{GHz}$, частота дискретизации - $5 \mathrm{GS} / \mathrm{s}$ ).

\section{Результаты экспериментов}

При регистрации спектров свечения алмазных образцов с помощью спектрометра OceanOptics, как и в работе [6], была зарегистрирована только ИКЛ. Зарегистрировать ИВЧ на уровне чувствительности регистрирующей аппаратуры не удалось. Тем не менее для обоих образцов в УФ диапазоне была обнаружена свободная от катодолюминесценции область спектра, располагающаяся между краем фундаментального поглощения и коротковолновым краем люминесценции. Поэтому можно было ожидать, что при увеличении чувствительности аппаратуры ИВЧ будет зарегистрировано в этой области спектра.

Для увеличения чувствительности в экспериментах использовалась схема регистрации ИВЧ, изображенная на рис. 2. С помощью этой схемы регистрации были получены осциллограммы интенсивности свечения алмазных образцов для разных длин волн. Также после интегрирования сигналов с ФЭУ было получено распределение энергии свечения по длинам волн для каждого образца, приведенное на рис. 3. Из рис. 3 следует, что в области 225-310 nm для природного алмаза и в области 225-350 nm для искусственного алмаза спектральная плотность энергии излучения увеличивается с уменьшением длины волны. В этих областях можно ожидать свечения экситонной полосы с максимумом на длине волны $235 \mathrm{~nm}$, однако время затухания люминесценции экситонной полосы в алмазе ІІа-типа составляет десятки наносекунд [8]. Регистрируемая длительность импульсов излучения образцов алмаза в этих областях не изменяется и соответствует временному разрешению ФЭУ $(2.2 \mathrm{~ns})$, т.е. на порядок меньше, чем время затухания люминесценции экситона. Поэтому можно утверждать, что свечение образцов в этих областях спектра соответствует ИВЧ. Также при этом наблюдается минимальная 

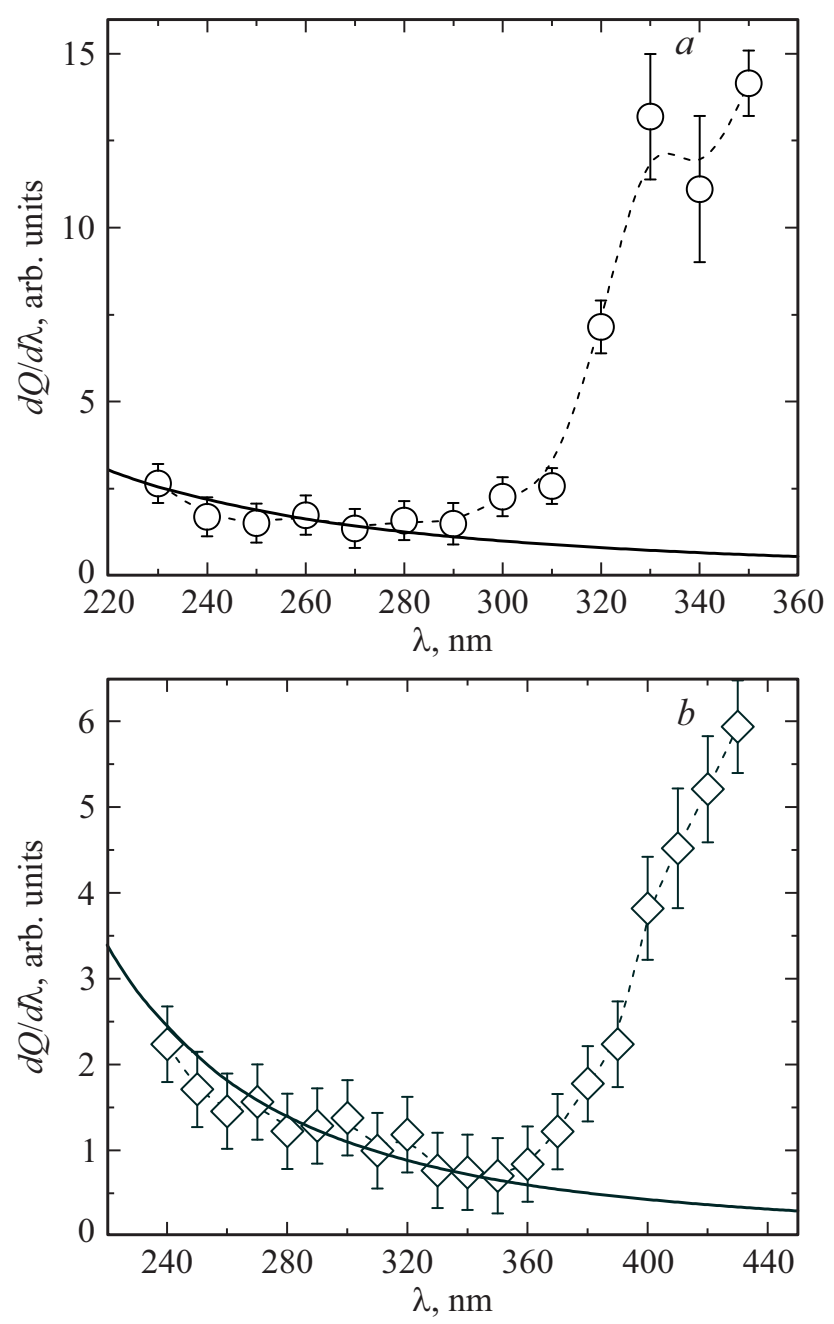

Рис. 3. Сглаженные экспериментальные зависимости спектральной плотности энергии излучения от длины волны (штриховые кривые) для природного (a) и искусственного $(b)$ алмазов, полученные с применением ФЭУ и монохроматора МДР-23, и расчетные кривые спектральной плотности энергии ИВЧ (сплошные кривые).

задержка импульсов излучения образцов алмаза относительно фронта импульса напряжения. Соответственно зарегистрированное оптическое излучение в данной области можно отнести к ИВЧ. Начиная с длины волны $310 \mathrm{~nm}$ для природного и $350 \mathrm{~nm}$ для искусственного алмаза наблюдается рост спектральной плотности энергии излучения и увеличение длительности импульсов излучения в несколько раз по мере увеличения длины волны. Кроме того, в этом диапазоне длин волн появляется задержка импульсов излучения относительно импульсов излучения, ассоциируемых с ИВЧ, которая плавно увеличивается до 4-5 ns.

\section{Постановка задачи}

Для того чтобы провести оценку соотношения энергий ИКЛ и ИВЧ, возбуждаемых внутри алмазных образ- цов при облучении их электронным пучком, необходимо вначале выделить в спектре свечения образцов составляющие, соответствующие ИКЛ и ИВЧ. Для этого можно рассчитать спектр ИВЧ, затем осуществить его привязку к экспериментальному спектру в области, свободной от люминесценции. Таким образом мы получим составляющую спектра, соответствующую ИВЧ. Если вычесть из суммарного спектра составляющую ИВЧ, то разность будет соответствовать спектру ИКЛ. Проинтегрировав по-отдельности спектры ИВЧ и ИКЛ, легко можно было бы найти соотношение их энергий. Однако в систему регистрации через линзу 9 (рис. 2) попадает лишь небольшая доля всей энергии как ИКЛ, так и ИВЧ, возбуждаемых в образцах. Поэтому для проведения дальнейших оценок следует выяснить величину этой доли.

Так как размеры образцов по сравнению с расстоянием до линзы малы (отличаются более чем на порядок), то в дальнейшем можно считать, что исследуемые образцы являются точечными источниками излучения [9]. Для расчета энергии ИКЛ, возбуждаемой внутри образцов, достаточно рассчитать, какую часть полного телесного угла составляет телесный угол с вершиной в центре алмазного образца и стягивающей поверхностью поверхностью линзы. Следует также учесть, что коэффициент отражения от грани образца составляет $\sim 17 \%$, и сделать поправку на коэффициент пропускания образца.

При расчете доли ИВЧ, попадающего в линзу, необходимо учитывать сразу несколько факторов. Во-первых, в отличие от люминесценции направление волнового фронта ИВЧ образует конус с вершиной в точке, совпадающей с мгновенным положением заряженной частицы (в нашем случае электрона). Угол между волновым вектором ИВЧ и направлением движения частицы удовлетворяет соотношению

$$
\cos \theta=u / v=c /(n v)=1 /(\beta n),
$$

где $u$ - скорость света в среде с показателем преломления $n, c$ - скорость света в вакууме, $v-$ скорость заряженной частицы, $\beta=v / c$.

Во-вторых, электрон с энергией до $1 \mathrm{MeV}$, двигаясь в веществе образца, теряет свою энергию в основном в процессе ионизации этого вещества. Ионизационные потери электронов описываются формулой БетеБлоха [10]

$$
\begin{aligned}
\left(-\frac{d E}{d x}\right)_{i}= & \frac{2 \pi e^{4} n_{e}}{m v^{2}}\left[\ln \frac{m v^{2} E}{2 I^{2}\left(1-\beta^{2}\right)}\right. \\
& -\ln 2\left(2 \sqrt{1-\beta^{2}}-1+\beta^{2}\right) \\
& \left.+1-\beta^{2}+\frac{1}{8}\left(1-\sqrt{1-\beta^{2}}\right)^{2}\right],
\end{aligned}
$$

где $E-$ кинетическая энергия электрона, $e-$ элементарный заряд электрона, $n_{e}-$ плотность электронов в веществе, $m$ - масса электрона, $v-$ скорость 
электрона, $I$ - средняя энергия возбуждения атомов вещества, через которое проходит электрон,

$$
\beta=\frac{v}{c}=\frac{\sqrt{\left(1+E /\left(m c^{2}\right)-1\right.}}{1+E /\left(m c^{2}\right)} .
$$

Поскольку электроны полностью тормозятся в образце, их энергия меняется от максимальной до нуля, и, следовательно, меняется угол $\theta$. При торможении меняется также спектр ИВЧ.

В-третьих, в реальном электронном пучке всегда имеется распределение электронов по энергиям, которое необходимо учитывать при расчете спектра ИВЧ.

В-четвертых, при движении в веществе образца электроны испытывают рассеяние, которое сказывается на ориентации конуса ИВЧ для каждого рассеянного электрона.

И наконец, в-пятых, при расчете спектров ИВЧ необходимо учитывать зависимость показателя преломления исследуемого материала от длины волны. Как правило, в различных источниках литературы (см., например, $[11,12])$ приводят показатели преломления вещества для определенной длины волны. Наиболее часто в литературе приводится значение показателя преломления $n$ для длины волны $D$-линии спектра натрия $589.3 \mathrm{~nm}$, измеренное при атмосферном давлении воздуха и температуре $20^{\circ} \mathrm{C}$ [13]. Этот показатель преломления обозначают $n_{D}$ или же просто $n$, подразумевая, что $n=n_{D}$. Если же учитывать дисперсию показателя преломления в широком спектральном диапазоне, то пороговая энергия электронов для возникновения ИВЧ будет ниже, чем пороговая энергия для возникновения ИВЧ, рассчитанная для $n_{D}$, и она будет соответствовать максимальной величине показателя преломления в области прозрачности этого вещества. Известно, что в области нормальной дисперсии вещества показатель преломления увеличивается с уменьшением длины волны, поэтому наибольшая величина показателя преломления будет соответствовать коротковолновой границе области прозрачности вещества.

Таким образом, для расчета спектров ИВЧ в широком спектральном диапазоне, где показатель преломления может существенно меняться, необходимо учитывать зависимость показателя преломления $n$ от длины волны $\lambda$. Так, для алмаза пороговая энергия возникновения ИВЧ, рассчитанная для показателя преломления $n_{D}$ составляет $\sim 50 \mathrm{keV}$, а для показателя преломления на длине волны, соответствующей коротковолновой границе области прозрачности ( $225 \mathrm{~nm}$, рис. 4, кривая 2$)$, составляет $\sim 39 \mathrm{keV}$.

\section{Оценка соотношения энергий ИКЛ и ИВЧ внутри алмазных образцов}

Потери энергии электрона на ИВЧ в интервале частот $d \omega$ можно определить, используя эффективную силу

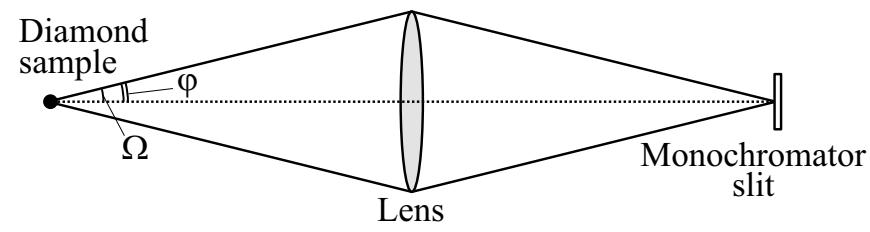

Рис. 4. Схематичное изображение телесного угла $\Omega$, образованного точечным источником излучения и поверхностью линзы.

радиационного торможения $F[14]$ :

$$
d F=\frac{e^{2}}{c^{2}}\left(1-\frac{1}{\beta^{2} n(\omega)^{2}}\right) \omega d \omega .
$$

Нетрудно перейти в выражении (2) от циклической частоты $\omega$ к длине волны $\lambda$ с помощью формулы $\omega=2 \pi c / \lambda$. Тогда спектральную плотность мощности ИВЧ $d P(\lambda) / d \lambda$ для одного электрона можно определить с помощью выражения

$$
d P(\lambda)=v d F=4 \pi^{2} e^{2} v\left(1-\frac{1}{\beta^{2} n(\lambda)^{2}}\right) \frac{d \lambda}{\lambda^{3}} .
$$

От выражения (3) можно перейти к спектральной плотности энергии излучения $d Q / d \lambda$, умножив выражение (3) на соответствующий временной интервал.

Чтобы рассчитать количественно спектр ИВЧ, умножаем выражение (1) на скорость электрона, и, решая это уравнение, находим изменение энергии электрона во времени. Подставляя получившиеся значения энергии тормозящихся в веществе электронов в формулу (3) (энергия входит в выражение для $\beta$ ) и умножая на соответствующий временной интервал, с учетом дисперсии показателя преломления $n(\lambda)$ определяем спектральную плотность энергии ИВЧ.

Для того чтобы оценить соотношение энергий ИКЛ и ИВЧ, возбуждаемых внутри алмазных образцов, были выполнены следующие действия.

1. Спектр электронного пучка был представлен пятью характерными точками.

2. Рассеяние электронов в анодной Ті-фольге и в алмазных образцах рассчитывалось с помощью программы ЕРНСА2 [15] методом Монте-Карло. В расчетах также учитывались ионизационные потериэнергии электронов по формуле Бете-Блоха (1). Анодная фольга и прилегающий к ней исследуемый образец разбивались по глубине (соответствующей полному пробегу электронов с определенной начальной энергией) на девять слоев, причем в качестве первого слоя выступала Тіфольга. После прохождении каждого слоя выводились распределения электронов по углам и по энергиям. Уже после прохождения первого слоя (Ті-фольги) угловое распределение слабо менялось даже для наибольшей начальной энергии электронов, поэтому разбиение на такое количество слоев можно считать вполне достаточным для проведения оценок. 


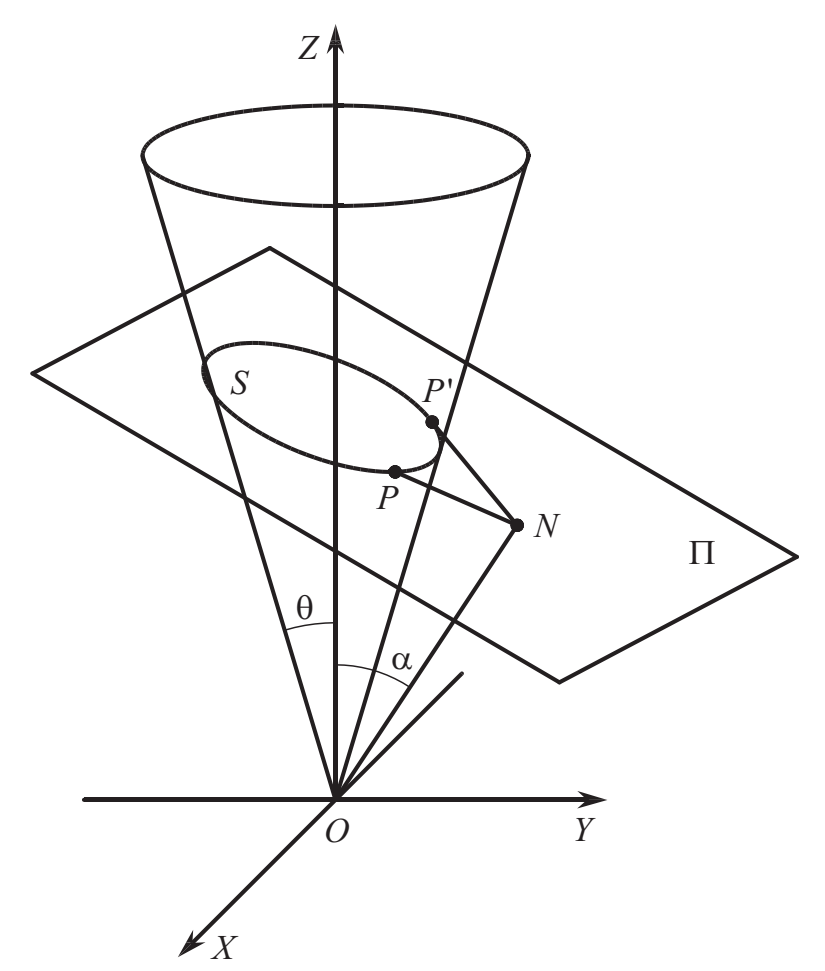

Pис. 5. Схематическое изображение конической поверхности, образованной волновым вектором ИВЧ, возникающего при движении электрона внутри алмазного образца, плоскости П, соответствующей поверхности образца, и эллипса $S$, образованного при их пересечении. Отрезок $O N$ перпендикулярен плоскости П.

3. Далее в среде Mathcad проводилась оценка доли ИВЧ, попадающей в линзу и затем в щель монохроматора (рис. 4).

Как уже говорилось, образцы алмаза можно представить как точечный источник излучения, поэтому задача сводится к определению доли излучения (ИВЧ и ИКЛ), попадающей в телесный угол $\Omega$, образованный алмазным образцом и линзой (рис. 4).

В каждом отдельном слое образца алмаза рассчитывалось ИВЧ с учетом распределения электронов по углам и энергиям (для оценки энергии ИВЧ в образце бралось значение энергии, соответствующее максимуму функции распределения в слое). ИВЧ, возникающее в каждом слое, суммировалось. Учитывалось, что для отдельно взятого электрона в алмазе конус черенковского излучения в каждый момент времени будет по-разному ориентирован в пространстве и только часть ИВЧ будет попадать в телесный угол $\Omega$ (рис. 4). Плоский угол $\varphi$ на рис. 4 равен $\sim 11^{\circ}$. Однако для того чтобы излучение, возникающее внутри образца, попадало в линзу под углом $\leq \varphi$, необходимо, чтобы внутри кристалла это излучение распространялось под углом не более $\sim 4^{\circ}$ к нормали, проведенной к поверхности образца.

Схематически распространение ИВЧ в алмазном образце изображено на рис. 5. Направление движения электрона совпадает с осью $Z$, а волновой вектор ИВЧ образует с осью $Z$ угол $\theta$. Для того чтобы определить, какая часть ИВЧ выходит из кристалла и попадает в линзу, необходимо прежде всего вычислить координаты точки $P-$ одной из двух точек пересечения эллипса $S$ и конуса ( $P$ и $P^{\prime}$, расположенных симметрично по отношению к плоскости $Z Y)$, внутри которого распространяется в образце часть ИВЧ, попадающая в линзу.

Координаты точки $P$ можно определить, решив приведенную ниже систему уравнений (4). Первое уравнение описывает коническую поверхность, образуемую волновым вектором ИВЧ. Второе уравнение описывает плоскость, соответствующую поверхности образца, из которой выходит излучение. Третье уравнение приравнивает к $\operatorname{tg}^{2}\left(4^{\circ}\right)$ отношение квадрата отрезка произвольной длины, изменяющегося вдоль отрезка $N P$, лежащего на плоскости $\Pi$, к $d^{2}(d=O N-$ расстояние между параллельными поверхностями образца).

$$
\begin{gathered}
\frac{x^{2}+y^{2}}{\operatorname{tg}^{2} \theta}=\frac{z^{2}}{1}, \\
(y-d \sin \alpha) \sin \alpha+(z-d \cos \alpha) \cos \alpha=0, \\
\frac{(y-d \sin \alpha)^{2}+x^{2}+(z-d \cos \alpha)^{2}}{d^{2}}=\left(\operatorname{tg} 4^{\circ}\right)^{2} .
\end{gathered}
$$

В любом сечении конической поверхности волнового вектора ИВЧ плоскостью, перпендикулярной оси $Z$, мощность ИВЧ будет равномерно распределена по азимутальному углу. Если провести плоскость, перпендикулярную оси $Z$, через точку $P$, то пересечение конической поверхности волнового вектора ИВЧ и этой плоскости образует окружность. Мощность ИВЧ при этом будет равномерно распределена по азимутальному углу. Зная координаты точки Р, нетрудно вычислить угол между осью $Y$ и радиусом, проведенным из центра окружности в точку $P$. Отношение этого угла к развернутому углу $\left(180^{\circ}\right)$ определяет часть ИВЧ, попадающую в линзу для ориентации конической поверхности волнового вектора ИВЧ, при которой одна из его образующих составляет с плоскостью П прямой угол. При такой ориентации в линзу попадает наибольшая часть энергии ИВЧ. При отклонении конической поверхности от этого положения часть ИВЧ, попадающая в линзу, уменьшается, и при отклонении образующей конической поверхности волнового вектора ИВЧ, лежащей в плоскости YZ, от нормали к плоскости П на угол порядка $\pm 4^{\circ}$ эта часть ИВЧ стремится к нулю. Поэтому для оценки сверху можно считать, что в среднем для всех электронов, излучение которых попадает в линзу, часть излучения ИВЧ, попадающая в линзу, не меньше половины от максимального значения этой части излучения. Количество таких электронов было рассчитано с использованием программы ЕРНСА 2.

Все эти вычисления были проведены для пяти различных начальных энергий, характеризующих распределение электронов пучка по энергиям, и суммированы для расчета всей энергии ИВЧ, попавшего в линзу. При этом учитывалось отражение от поверхности образца 
и его пропускание. Расчет показал, что доля ИВЧ, попадающая в линзу, от всего ИВЧ, возникающего внутри плоскопараллельной пластины алмаза, составляет $\sim 0.2 \%$. Так как при проведении экспериментов электронный пучок полностью поглощался в образцах, то для существенного увеличения этой доли необходимо использовать алмазные образцы другой формы.

4. Заключительным шагом является оценка соотношения энергий ИКЛ и ИВЧ в спектре свечения алмазов при возбуждении их внутри алмазных образцов в процессе облучения электронным пучком. Как говорилось ранее, предварительно рассчитанный спектр ИВЧ (с учетом пропускания образца и отражения излучения от его граней) совмещался со спектром, полученным с помощью монохроматора и ФЭУ (рис. 3). Была также сделана „сшивка“ спектра, полученного с помощью спектрометра OceanOptics, со спектром, полученным с помощью монохроматора и ФЭУ. Затем по описанной в предыдущем разделе схеме путем вычитания спектра ИВЧ и последующим интегрированием получившегося спектра ИКЛ и спектра ИВЧ, и принимая во внимание, что только часть излучения ИКЛ и ИВЧ попадает в линзу, была проведена оценка соотношения энергий ИВЧ и ИКЛ внутри алмазных образцов. Расчеты показали, что в диапазоне длин волн $240-750 \mathrm{~nm}$ энергия ИВЧ, возбуждаемого в алмазных образцах, составляет не более $\sim 17 \%$ и $\sim 0.4 \%$ от энергии ИКЛ для синтетического и природного алмазов соответственно (погрешность измерения энергии ИКЛ и ИВЧ составила 13\% и $\sim 10 \%$ соответственно). Столь большая разница в процентном отношении энергии ИВЧ к энергии ИКЛ для синтетического и природного алмазов объясняется тем, что для синтетического алмаза энергия ИКЛ более чем на порядок меньше, чем для природного.

\section{Заключение}

Проведенные на основе экспериментальных спектров оценки позволяют сделать вывод, что при облучении алмазных образцов пучком электронов с энергией до $200-300 \mathrm{keV}$ основную долю возбуждаемого излучения составляет ИКЛ. Для регистрации ИВЧ следует использовать свободную от полос катодолюминесценции спектральную область в УФ диапазоне, располагающуюся между краем фундаментального поглощения и коротковолновым краем люминесценции. Интенсивность ИВЧ, выходящего из алмазного радиатора, можно значительно увеличить. Для этого форма алмазных радиаторов должна быть такой, чтобы концентрировать ИВЧ на выходе из радиатора и минимизировать влияние полного внутреннего отражения на выходящее из радиатора излучение.

\section{Благодарности}

Работа выполнена при поддержке Российского научного фонда (проект № 18-19-00184).

\section{Конфликт интересов}

Авторы заявляют, что у них нет конфликта интересов.

\section{Список литературы}

[1] Bagnato F. et al. // Plasma Phys. Control. Fusion. 2018. V. 60. P. 115010. doi 10.1088/1361-6587/aae0b3

[2] Sadowski M.J. // Nukleonika. 2011. V. 56. N 2. P. 85.

[3] Jakubowski L. et al. // Nukleonika. 2012. V. 57. N 2. P. 177.

[4] Jakubowski L. et al. // Rev. Sci. Instr. 2010. V. 81. N 1. P. 013504. doi 10.1063/1.3280221

[5] Соломонов В.И., Михайлов С.Г. Импульсная катодолюминесценция и ее применение для анализа конденсированных веществ. Екатеринбург: Издательство УрО РАН, 2003. $182 \mathrm{c}$.

[6] Sorokin D.A. et al. // J. Appl. Phys. 2017. V. 122. N 15. P. 154902. doi 10.1063/1.4996965

[7] Загулов Ф.Я., Котов А.С., Шпак В.Г., Юрике Я.Я., Яландин М.И. // ПТЭ. 1989. № 2. С. 146.

[8] Takiyama K., Abd-Elrahman M.I., Fujita T., Oda T. // Sol. Stat. Commun. 1996. V. 99. N 11. P. 793.

[9] Сапожников Р.А. Теоретическая фотометрия. М.: Энергия, 1977. $264 \mathrm{c}$.

[10] Бекман И.Н. Ядерная физика. Курс лекций. М.: МГУ, 2010. $511 \mathrm{c}$.

[11] Альбиков 3.А., Веретенников А.И., Козлов О.В. Детекторы импульсного ионизирующего излучения. М.: Атомиздат, 1978. $176 \mathrm{c.}$

[12] Беспалов В.И. Взаимодействие ионизирующих излучений с веществом. Учебное пособие. Томск: Томский политехнический университет, 2008. 368 с.

[13] Строганова Е.А., Паршина И.Н., Киекпаев М.А., Пономарева П.А. Органическая химия. Ч. 2. Методы выделения, очистки и идентификации органических соединений: практикум. Оренбург: ОГУ, 2013. 126 с.

[14] Ландау Л.Д., Лифиии Е.М. Теоретическая физика. Т. 8. Электродинамика сплошных сред. М.: Наука, 1982. 621 с.

[15] Беспалов В.И. // Изв. вузов. Физика. 2000. № 4. С. 159. 